\title{
SOME THEOREMS ON CONTINUA ${ }^{1}$
}

\author{
EDWIN W. MILLER
}

The following theorem, proved by A. Mullikin in her thesis, ${ }^{2}$ has been used extensively in certain point set theoretic investigations.

Theorem. If $C$ is a continuum and $F_{1}$ and $F_{2}$ are closed, mutually exclusive and nonvacuous subsets of $C$, then there exists a component of $C-\left(F_{1}+F_{2}\right)$ which has a limit point in $F_{1}$ and a limit point ${ }^{3}$ in $F_{2}$.

In $\S 1$ we shall obtain a theorem (Theorem 1 ) which represents a strengthening of Mullikin's result. The theorem, in this stronger form, has numerous applications. We shall discuss some of these applications in $\$ 2$ and $\S 3$.

1. A stronger form of Mullikin's theorem. We prove the following theorem:

THEOREM 1. Under the hypotheses of Mullikin's theorem, there exists a constituent ${ }^{4}$ of $C-\left(F_{1}+F_{2}\right)$ which has a limit point in $F_{1}$ and a limit point in $F_{2}$.

PRoof. Let $\left\{G_{n}\right\}$ and $\left\{H_{n}\right\}$ denote monotonic decreasing sequences of open sets which close down upon $F_{1}$ and $F_{2}$ respectively. We may suppose that $G_{1}$ and $H_{1}$ are so chosen that $\bar{G}_{1} \cdot \bar{H}_{1}=0$. Now, by Mullikin's theorem, there is a connected subset of $C-C \cdot\left(\bar{G}_{n}+\bar{H}_{n}\right)$ which has a limit point in $\bar{G}_{n}$ and a limit point in $\bar{H}_{n}$. The closure of such a connected set is a subcontinuum of $C$ which "extends" from $\bar{G}_{n}$ to $\bar{H}_{n}$. If, then, we denote by $Q_{n}$ the set of all points of $C-C \cdot\left(G_{n}+H_{n}\right)$ which lie on subcontinua of $C$ which extend (in the above indicated sense) from $\bar{G}_{n}$ to $\bar{H}_{n}$, we have $Q_{n} \neq 0$.

\footnotetext{
${ }^{1}$ Presented to the Society, April 10,1937, under the title On a theorem due to A. Mullikin.

${ }^{2}$ Certain theorems relating to plane connected point sets, Transactions of this Society, vol. 24 (1922), pp. 144-162.

${ }^{3}$ In Mullikin's statement of this theorem, $C$ is a bounded plane continuum. It is clear, however, that her proof applies if $C$ is any continuum in a compact metric space. In the present paper, all point sets under consideration are understood to be embedded in a compact metric space, except when the contrary is expressly stated. If $M$ is a point set and $p$ is a point of $M$, then the maximal connected subset of $M$ which contains $p$ is called the component of $M$ determined by $p$.

${ }^{4} \mathrm{~A}$ set of points $K$ is said to be strongly connected, or to be a semicontinuum, if every pair of points of $K$ lies on some continuum contained in $K$. If $M$ is a point set and $p$ is a point of $M$, then the maximal strongly connected subset of $M$ which contains $p$ is called the constituent of $M$ determined by $p$.
} 
By the same argument it follows that any subcontinuum of $C$ which extends from $\bar{G}_{n}$ to $\bar{H}_{n}$ contains a continuum which extends from $\bar{G}_{n-1}$ to $\bar{H}_{n-1}$. This continuum, in turn, contains a continuum which extends from $\bar{G}_{n-2}$ to $\bar{H}_{n-2}$, and so on, so that for any $n$ we have $\prod_{k=1}^{n} Q_{k} \neq 0$.

It will now be shown that for every $n$, the set $Q_{n}$ is closed. Let $p$ be a limit point of any sequence of points $p_{1}, p_{2}, \cdots, p_{m}, \cdots$ of $Q_{n}$. Each point $p_{m}$ belongs to a subcontinuum of $C$ extending from $\bar{G}_{n}$ to $\bar{H}_{n}$. If $p$ is not a point of any of these continua, there will exist, according to a theorem due to Janiszewski, ${ }^{5}$ a limit continuum, again a subcontinuum of $C$, which contains $p$ and extends from $\bar{G}_{n}$ to $\bar{H}_{n}$.

Let $A_{n}=\prod_{k=1}^{n} Q_{k}$. It is clear that the sets $A_{1}, A_{2}, \cdots, A_{n}, \cdots$ form a monotonic decreasing sequence of closed, compact and nonvacuous sets. There will exist, according to a well known theorem due to Cantor, at least one point $p$ common to all the sets $A_{n}$. Clearly, $p \varepsilon \prod_{n=1}^{\infty} Q_{n}$. From the definition of $Q_{n}$ it is obvious that the constituent of $C-\left(F_{1}+F_{2}\right)$ which such a point $p$ determines has a limit point in $F_{1}$ and a limit point in $F_{2}$.

2. Applications to continua in the plane. In this section we shall use Theorem 1 to obtain certain results regarding the common boundary of two plane domains.

THEOREM 2. If $B$ is a bounded plane continuum which is the common boundary of two or more domains, and if $C$ is a closed proper subset of $B$ which contains at least two points, then there is a constituent of $B-C$ which has at least two limit points in $C$.

Proof. If $C$ is not a continuum, then $C=C_{1}+C_{2}$, where $C_{1}$ and $C_{2}$ are closed, nonvacuous sets, and $C_{1} \cdot C_{2}=0$. Then, by Theorem 1 , there is a constituent of $B-C$ which has a limit point in $C_{1}$ and a limit point in $C_{2}$, and therefore two limit points in $C$.

Suppose now that $C$ is a continuum. We may distinguish two cases according as $B$ is an indecomposable or a decomposable continuum.

Suppose that $B$ is an indecomposable continuum. Then, since $C$ is a proper subcontinuum of $B$, it lies entirely in some composant of $B$. Let $K$ be any other composant of $B$. Then $K \subset B-C$. Now, it is well known that any composant of an indecomposable continuum is dense in that continuum. ${ }^{6}$ Therefore every point of $C$ is a limit point of $K$. But clearly, $K$ is a constituent of $B-C$.

${ }^{5}$ Z. Janiszewski, Sur les continus irréductibles entre deux points, Journal de l'Êcole Polytechnique, (2), vol. 16 (1912), p. 98, Theorem 1.

${ }^{6}$ See Z. Janiszewski and C. Kuratowski, Sur les continus indécomposables, Fundamenta Mathematicae, vol. 1 (1920), p. 221, Theorem 8. 
Suppose now that $B$ is a decomposable continuum. In this case, according to a theorem proved independently by $\mathrm{C}$. Kuratowski ${ }^{7}$ and W. A. Wilson, ${ }^{8}$ the continuum $B$ is the sum of two continua $K$ and $L$, such that $K \cdot L=M+N$, where $M$ and $N$ are closed, mutually exclusive and nonvacuous sets such that $K$ and $L$ are irreducible between $M$ and $N$.

It is easily seen that there are just four essentially different cases here:

1. $C \subset K-(M+N)$,

2. $C \cdot M \neq 0$ and $C \cdot N=0$,

3. $C=K$,

4. $C \supset K$ and $C \neq K$.

Case 1. Let $K_{1}$ be a constituent of $K-C$ determined by a point of $M$, and $K_{2}$ a constituent of $K-C$ determined by a point of $N$. Then, from a theorem due to Janiszewski, ${ }^{9}$ the constituents $K_{1}$ and $K_{2}$ have each at least one limit point in $C$. Then obviously the set $K_{1}+K_{2}$ has at least two limit points in $C$, for otherwise $\overline{K_{1}+K_{2}}$ would be a proper subcontinuum of $K$ which contains points of $M$ and points of $N$. It follows that $L+K_{1}+K_{2}$ is contained in a constituent of $B-C$ which has at least two limit points in $C$.

Case 2. Let $x$ be any point of $N$. Denote by $P$ the constituent of $K-C$ determined by $x$, and by $Q$ the constituent of $L-C$ determined by $x$. If either $P$ or $Q$ has as many as two limit points in $C$, then a constituent of $B-C$ of the desired sort is determined. Assume that $P$ has just one limit point, $y$, in $C$, and that $Q$ has just one limit point, $z$, in $C$. Now if $y=z$, then $y$ must be a point of $M$, and it follows easily that either $\bar{P}$ is a proper subcontinuum of $K$ which contains a point of $M$ and a point of $N$, or $\bar{Q}$ is a proper subcontinuum of $L$ which contains a point of $M$ and a point of $N$. Hence $y \neq z$, and $P+Q$ is contained in a constituent of $B-C$ which has at least two limit points in $C$.

Case 3. By Theorem 1 , there is a constituent of $L-(M+N)$ which has a limit point in $M$ and a limit point in $N$. This is the constituent sought.

Case 4 . In this case $C \cdot L$ is a closed, nonvacuous and proper subset of $L$. It cannot be a continuum since $L$ is irreducible between $M$ and $N$, and $C \cdot L$ contains both $M$ and $N$. Accordingly, $C \cdot L=C_{1}+C_{2}$,

${ }^{7}$ C. Kuratowski, Sur la séparation des ensembles, Fundamenta Mathematicae, vol. 12 (1928), p. 235, Theorem 7.

${ }^{8} \mathrm{~W}$. A. Wilson, On bounded regular frontiers in the plane, this Bulletin, vol. 34 (1928), p. 86, Theorem 6.

${ }^{9} \mathrm{Z}$. Janiszewski, loc. cit., Theorem 4. 
where $C_{1}$ and $C_{2}$ are closed, mutually exclusive, nonvacuous subsets of $L$. Hence Theorem 1 applies and a constituent of the desired sort is obtained.

THEOREM 3. If $D$ is a bounded and connected plane domain and $M=\sum_{n=1}^{\infty} M_{n}$, where $M_{m} \cdot M_{n}=0$ if $m \neq n$, and each set $M_{n}$ is a continuum which does not cut the plane and which lies, except for one point at most, entirely in $D$, then $D-D \cdot M$ is connected.

Proof. Let us assume that $W=D-D \cdot M$ is not connected. Then, according to a theorem of Knaster and Kuratowski, ${ }^{10}$ there is a continuum $K$ which cuts the plane between two points $a$ and $b$ of $W$ and lies entirely in the complement of $W$. Now, if $H$ denotes the boundary of $D$, it is easily proved that the closed set $H+K \cdot D$ cuts the plane between $a$ and $b$. It follows by theorems due to Mazurkiewicz ${ }^{11}$ and Kuratowski ${ }^{12}$ that $H+K \cdot D$ contains a continuum $L$ which is the common boundary of two domains which contain $a$ and $b$ respectively.

Now the set $L \cdot H$ must contain more than one point-in fact, it must contain a nondenumerable infinity of points. Otherwise, as follows easily from our hypotheses on the continua $M_{n}$, the equation $L=L \cdot H+\sum_{n=1}^{\infty} L \cdot M_{n}$ determines a decomposition of the bounded continuum $L$ into a denumerable infinity of closed, mutually exclusive and nonvacuous sets. This is impossible as Sierpiński has shown. ${ }^{13}$

Since the closed set $L \cdot H$ contains more than one point and is a proper subset of $L$, we may apply Theorem 2 . There exists, then, a constituent $Q$ of $L-L \cdot H$ which has at least two limit points in $H$. Now $Q$ must contain points of two different continua $M_{n}$, since each continuum $M_{n}$ contains at most one point of $H$. Then $Q$, and therefore $M$, contains a continuum which contains points of two different sets $M_{n}$. But this contradicts Sierpiński's theorem.

3. Extensions of Sierpinski's theorem. The theorem of Knaster and Kuratowski employed in the proof of Theorem 3 is of considerable importance in establishing the existence of different sorts of con-

${ }_{10} \mathrm{~B}$. Knaster and C. Kuratowski, Sur les ensembles connexes, Fundamenta Mathematicae, vol. 2 (1921), p. 233, Theorem 37.

${ }^{11} \mathrm{~S}$. Mazurkiewicz, Sur un ensemble $G_{\delta}$, punctiforme, qui n'est pas homéomorphe avec aucun ensemble linéaire, Fundamenta Mathematicae, vol. 1 (1920), p. 63, Theorem 1.

${ }^{12} \mathrm{C}$. Kuratowski, Sur les coupures irréductibles du plan, Fundamenta Mathematicae, vol. 6 (1925), p. 133, Theorem 3.

${ }^{13} \mathrm{~W}$. Sierpinski, Un théorème sur les continus, Tôhoku Mathematical Journal, vol. 13 (1918), pp. 300-303. 
nected sets. ${ }^{14}$ The content of the theorem is that if we construct a bounded plane set in such a way that no continuum in its complement separates any two points of the set, then we may be sure that the set constructed is connected. From this point of view the theorem of Sierpinski employed in the proof of Theorem 3 is of importance. From Sierpiński's theorem and the theorem of Knaster and Kuratowski it easily follows, for example, that if we delete from the interior of a circle a set which is the sum of a denumerable infinity of mutually exclusive closed sets, no one of which cuts the plane, then the set of points remaining is connected. It is clear that extensions of Sierpiński's theorem will yield us more general procedures for the construction of connected sets. In this section we shall accordingly establish a few theorems relating to the following general question. If $A$ and $M$ are point sets in a compact metric space and $M=\sum_{n=1}^{\infty} M_{n}$, where the $M_{n}$ are mutually exclusive closed sets, under what circumstances will $A+M$ fail to contain an $M$-join, that is, a continuum which contains a point of each of two different sets $M_{n}$ ?

Theorem 4. Let $P$ be a closed punctiform set and let $M=\sum_{n=1}^{\infty} M_{n}$, where (1) the sets $M_{n}$ are mutually exclusive and closed, and (2) every component of every set $M_{n}$ has at most one point in common with $P$. Then $M+P$ contains no $M$-join.

Proof. Suppose the contrary, and let $C$ denote an $M$-join contained in $M+P$. Then from Sierpinski's theorem, $C \cdot P$ is nondenumerable. (For our purpose it is enough that $C \cdot P$ contains more than one point.) We may write $C \cdot P=C_{1}+C_{2}$, where $C_{1}$ and $C_{2}$ are closed, mutually exclusive and nonvacuous. By Theorem 1 , there is a constituent $Q$ of $C-C \cdot P$ which has a limit point in $C_{1}$ and a limit point in $C_{2}$. From condition (2), the semicontinuum $Q$ must contain points of two different sets $M_{n}$. Therefore $Q$ contains an $M$-join. But this contradicts Sierpiński's theorem.

Theorem 5. Let $P$ be a closed punctiform set and $M=\sum_{n=1}^{\infty} M_{n}$, where (1) the sets $M_{n}$ are mutually exclusive and closed, and (2) there exists an $\epsilon>0$ such that $K$ is of diameter greater than $\epsilon$ if $K$ contains more than one point of $P$ and is a continuum contained in any $M_{n}$. Then $M+P$ contains no $M$-join.

Proof. Suppose the contrary, and let $C$ denote an $M$-join contained in $M+P$. Then, by Sierpiński's theorem, $C \cdot P-C \cdot M \neq 0$. Let

${ }^{14}$ See Knaster and Kuratowski, loc. cit. See also P. M. Swingle, Two types of connected sets, this Bulletin, vol. 37 (1931), pp. 254-258, and E. W. Miller, Concerning biconnected sets, Fundamenta Mathematicae, vol. 29 (1937), pp. 123-133. 
$x$ be a point of $C \cdot P-C \cdot M$. Then, by a theorem due to Janiszewski, ${ }^{15}$ $C$ contains a subcontinuum $C_{1}$ which contains $x$ and is of diameter less than $\epsilon$. Clearly, $C_{1}$ is an $M$-join. However, it is clear from the hypotheses of the present theorem that the sets $C_{1} \cdot M_{n}$ satisfy the hypotheses of Theorem 4 , so that the existence of $C_{1}$ contradicts Theorem 4.

THEOREM 6. Let $M=\sum_{n=1}^{\infty} M_{n}$, where the sets $M_{n}$ are mutually exclusive and closed. Let $P=\sum_{k=1}^{\infty} P_{k}$, where the sets $P_{k}$ are punctiform and closed. If for every $k$, the set $M+P_{k}$ contains no $M$-join, then $M+P$ contains no $M$-join.

We omit the proof of Theorem 6 since it closely follows the lines of the proof of Sierpiński's theorem.

In the present connection the following question is a natural one. Let $M=\sum_{n=1}^{\infty} M_{n}$, where the sets $M_{n}$ are mutually exclusive and closed, and let $N=\sum_{k=1}^{\infty} N_{k}$, where the sets $N_{k}$ are mutually exclusive and closed. If for every $k$ the set $M+N_{k}$ contains no $M$-join, will it be true that $M+N$ contains no $M$-join? The following example shows that this is not necessarily the case.

On a linear interval $(a, b)$ take a nowhere dense perfect set whose first point on $(a, b)$ is $a$ and whose last point is $b$. This will be the set $N_{1}$. Now on each interval $\left(a^{\prime}, b^{\prime}\right)$ contiguous to $N_{1}$ take a nowhere dense perfect set whose first point on $\left(a^{\prime}, b^{\prime}\right)$ is $a^{\prime}$ and whose last point is $b^{\prime}$. These perfect sets will be our sets $M_{n}$. We take as our sets $N_{2}, N_{3}, N_{4}, \cdots$ the various closed intervals contiguous to the various sets $M_{n}$. It is easily seen that the conditions mentioned above are satisfied, and yet $M+N$ is the entire linear interval $(a, b)$. For future reference it may be noticed here that $N_{1}$ has a point in common with infinitely many, in fact, with all of the sets $M_{n}$, and that there is a nondenumerable infinity of components of $N_{1}$ which have no point in common with $M$.

LEmma. If (1) $M=\sum_{n=1}^{\infty} M_{n}$, where the sets $M_{n}$ are mutually exclusive, closed and nonvacuous, and (2) $N=\sum_{k=1}^{\infty} N_{k}$, where the sets $N_{k}$ are mutually exclusive, closed and nonvacuous, and (3) for every $k$ the set $M+N_{k}$ contains no $M$-join, then $C \cdot M_{n} \neq 0$ for infinitely many indices $n$, if $C$ is an $M$-join contained in $M+N$.

Proof. Suppose that $C \cdot M_{n} \neq 0$ for only a finite number of indices $n$. To simplify the notation, let us suppose that $C \cdot M_{n} \neq 0$ for $n=1,2, \cdots, r$, and $C \cdot M_{n}=0$ for $n>r$. By Theorem 1 , there is a constituent $Q$ of $C-C \cdot\left(M_{1}+M_{2}+\cdots+M_{r}\right)$ which has a limit point

${ }^{15} \mathrm{Z}$. Janiszewski, loc. cit., Theorem 4. 
in $M_{1}$ and a limit point in $M_{2}+\cdots+M_{r}$. We have, of course, $Q \cdot M=0$. Therefore $Q \subset N$. By Sierpiński's theorem, $Q$ must be a subset of some one set $N_{k}$. But this set $N_{k}$ would then contain an $M$-join. As this contradicts condition (3), the result follows.

THEOREM 7. If conditions (1), (2) and (3) of the preceding lemma hold, and if (4) for every $k, M_{n} \cdot N_{k} \neq 0$ for at most one $n,{ }^{16}$ then $M+N$ contains no M-join.

Proof. Let us assume that $M+N$ contains an $M$-join $C$. We will first show that $C$ must contain an $M$-join $C_{1}$ such that $C_{1} \cdot\left(M_{1}+N_{1}\right)$ $=0$.

By the preceding lemma and by condition (4), there is a positive integer $j$ such that $C \cdot M_{j} \neq 0$ and $M_{j} \cdot\left(M_{1}+N_{1}\right)=0$. If $C \cdot\left(M_{1}+N_{1}\right) \neq 0$, let $K$ be a subcontinuum of $C$ irreducible between ${ }^{17} M_{1}+N_{1}$ and $M_{j}$.

Case 1. Suppose $K$ is a decomposable continuum. Then $K=K_{1}+K_{2}$, where $K_{1}$ and $K_{2}$ are proper subcontinua of $K, K_{1} \supset K \cdot\left(M_{1}+N_{1}\right)$, $K_{1} \cdot M_{j}=0, K_{2} \supset K \cdot M_{j}$ and $K_{2} \cdot\left(M_{1}+N_{1}\right)=0$. By Theorem 1 there is a constituent $Q$ of $K-K \cdot\left(M_{1}+N_{1}+M_{j}\right)$ which has a limit point in $M_{j}$ and a limit point in $M_{1}+N_{1}$. From the conditions of the present theorem and from Sierpinski's theorem it follows easily that $Q \cdot M \neq 0$. Furthermore we have $Q \cdot K_{2} \neq 0$. Then, clearly, $Q+K_{2}$ contains an $M$-join $C_{1}$ such that $C_{1} \cdot\left(M_{1}+N_{1}\right)=0$.

Case 2. Suppose that $K$ is an indecomposable continuum. Since $K$ is irreducible between $M_{1}+N_{1}$ and $M_{j}$, there exists a composant $S_{1}$ of $K$ such that $S_{1} \cdot\left(M_{1}+N_{1}\right) \neq 0$ and $S_{1} \cdot M_{j}=0$. For the same reason there exists a composant $S_{2}$ of $K$ such that $S_{2} \cdot M_{j} \neq 0$ and $S_{2} \cdot\left(M_{1}+N_{1}\right)=0$. If $S_{2}$ contains an $M$-join $C_{1}$, then $C_{1} \cdot\left(M_{1}+N_{1}\right)=0$. We will suppose, then, that $S_{2}$ contains no $M$-join. Then, since $S_{2}$ is dense in $K$, the sets $N_{1}$ and $M_{n}$ (for $n \neq j$ ) are all nowhere dense in $K$. Likewise $M_{j}$ is nowhere dense in $K$ since $S_{1}$ is dense in $K$. Therefore, in virtue of the theorem of Baire, there must exist a positive integer $h \neq 1$ such that $N_{h}$ is dense in some region of $K$. It follows that there exists a continuum $I$ in $S_{2}$ such that $L \cdot M_{j} \neq 0$ and $L \cdot N_{h} \neq 0$. Suppose $M_{j} \cdot N_{h}=0$. Then, by Theorem 1 , there is a constituent $S$ of $L-L \cdot\left(N_{h}+M_{j}\right)$ which has a limit point in $N_{h}$ and a limit point

${ }^{16}$ Condition (4) can be replaced by $\left(4^{\prime}\right)$ : For each given $k$, either (a) $M_{n} \cdot N_{k} \neq 0$ for at most a finite number of indices $n$, or (b) at most a countable infinity of components of $N_{k}$ fail to have a point in common with $M$. In fact, it is not hard to show that (1), (2), (3), and (4') imply that $N$ can be re-expressed as the sum of a countable infinity of sets in such a way that for these new sets $N_{k}$, conditions (2), (3) and (4) are satisfied.

${ }^{17}$ Z. Janiszewski, loc. cit., p. 109, Theorem 1. 
in $M_{j}$. It is easily shown that $S \cdot M \neq 0$. But then $L$, and therefore $S_{2}$, would contain an $M$-join. We may assume, then, that $M_{j} \cdot N_{h} \neq 0$. Now, there is a constituent $Q$ of $K-K \cdot\left(M_{1}+N_{1}+M_{j}\right)$ which has a limit point in $M_{1}+N_{1}$ and a limit point in $M_{j}$. We will have $Q \cdot M \neq 0$. Now $Q$ must contain an $M$-join, for otherwise, $Q \cdot M=Q \cdot M_{r}$ where $r \neq j$, and since $Q$ is dense in $K$, it would follow by the argument just used that $M_{r} \cdot N_{h} \neq 0$. But this is impossible by condition (4). This completes the proof that $C$ must contain an $M$-join $C_{1}$ such that $C_{1} \cdot\left(M_{1}+N_{1}\right)=0$.

In the same way as above, we now can prove that $C_{1}$ contains an $M$-join $C_{2}$ such that $C_{2} \cdot\left(M_{2}+N_{2}\right)=0$. In general, we have $C_{k-1} \supset C_{k}$, where $C_{k}$ is an $M$-join such that $C_{k} \cdot\left(M_{k}+N_{k}\right)=0$. By the Cantor product theorem, there is at least one point $p$ common to all the continua $C_{k}$. But this is impossible, since $p$ must belong to $M+N$ and yet does not belong to $M_{k}+N_{k}$ for any $k$. This completes the proof of our theorem.

UNIVERSITY OF MichigAN 